\title{
Innovative strategies to treat skin wounds with mangiferin: fabrication of transfersomes modified with glycols and mucin
}

\author{
Mohamad Allaw $\ddagger, 1$, Maria Pleguezuelos-Villa $\ddagger, 2$, Maria Letizia Manca*,1 (iD, Carla Caddeo, \\ Matteo Aroffu1, Amparo Nacher ${ }^{2,3}$, Octavio Diez-Sales ${ }^{2,3}$, Amparo Ruiz Saurí4, Elvira \\ Escribano Ferrer ${ }^{5}$, Anna Maria Fadda ${ }^{1}$ \& Maria Manconi ${ }^{1}$ \\ ${ }^{1}$ Department of Scienze della Vita e dell'Ambiente, University of Cagliari, Via Ospedale 72, Cagliari, 09124, Italy \\ ${ }^{2}$ Department of Pharmacy \& Pharmaceutical Technology \& Parasitology, University of Valencia, Burjassot, Vicente Andrés Estellés \\ $s / n$, Valencia, 46100 , Spain \\ ${ }^{3}$ Institute of Molecular Recognition \& Technological Development, Inter-University Institute from Polytechnic University of Valencia \\ \& University of Valencia, Burjassot, 46100, Spain \\ ${ }^{4}$ Department of Pathology, University of Valencia, Avda Blasco Ibañez 17, Valencia, 46010, Spain \\ ${ }^{5}$ Biopharmaceutics \& Pharmacokinetics Unit, Institute for Nanoscience \& Nanotechnology, University of Barcelona, \\ Barcelona, 08193, Spain \\ *Author for correspondence: Tel.: +39 070675 8582; Fax: +39 070675 8553; mlmanca@unica.it \\ ¥Authors contributed equally
}

Aim: The moisturizing properties of glycerol, the penetration enhancing capability of propylene glycol and the bioadhesive properties of mucin were combined to improve the carrier capabilities of transfersomes and the efficacy of mangiferin in the treatment of skin lesions. Materials \& methods: Mangiferin was incorporated in transfersomes and glycoltransfersomes, which were also modified with mucin. The physico-chemical features were assessed, along with the efficacy against oxidative stress and skin wounds in vitro and in vivo. Results: Glycoltransfersomes promoted the deposition of mangiferin in epidermis and dermis, protected fibroblasts from oxidative stress and stimulated their proliferation. The wound healing and anti-inflammatory efficacy of glycoltransfersomes were confirmed in vivo. Conclusion: Results confirmed the potential of glycoltransfersomes in preventing/treating of skin lesions.

First draft submitted: 19 March 2020; Accepted for publication: 21 May 2020; Published online: 17 July 2020

Keywords: glycols $\bullet$ mangiferin $\bullet$ oxidative damage $\bullet$ skin delivery $\bullet$ transfersomes $\bullet$ wound healing

Traditional folk medicine still provides suitable therapies for millions of people worldwide through the use of natural compounds with therapeutic or beneficial properties. Given that, plant extracts and natural molecules have been attracting the interest of researchers [1]. Recent studies have underlined the importance of nanomedicine in potentiating the efficacy of natural compounds by the enhancement of their pharmacokinetics and bioavailability at the target tissue [2-5]. Pharmaceutical nanotechnologies can innovate traditional health-promoting preparations, helping to overcome challenges and ensuring their translation from bench to clinical application. Among the different nanosystems explored over the years, phospholipid vesicles represent one of the most valuable and versatile systems, especially for skin delivery, owing to their structure, biocompatibility and similarity to skin components [6-9]. Over the last three decades, new kinds of phospholipid vesicles have been proposed, such as ultra-deformable vesicles, namely transfersomes, for the transdermal delivery of drugs [10]. Transfersomes are more elastic and deformable than conventional liposomes, due to the presence of an edge activator, which is a surfactant or another molecule capable of modifying the assembly of the bilayer. Other phospholipid vesicles have been proposed to improve dermal and transdermal delivery of drugs [8]. Ethosomes are soft vesicles prepared by using a blend of ethanol and water as hydrating medium of phospholipids [11-13]. Transethosomes have been developed by combining ethanol and an edge activator to improve the deformability of vesicles [14]. Penetration 
enhancer-containing vesicles have been formulated to facilitate skin delivery of bioactive molecules: they contain a penetration enhancer (e.g., propylene glycol or ethylene glycol) that intercalates the bilayer or blends in the aqueous medium $[15,16]$. Glycerosomes have also been formulated for skin delivery by using mixtures of water and glycerol [17].

All these vesicles have been tested as carriers for drugs, natural molecules and extracts with antioxidant and antiinflammatory properties, showing promising capabilities in the treatment of skin disorders [18-21]. In particular, the delivery of natural bioactive molecules, such as curcumin, resveratrol, quercetin, baicalin and mangiferin in phospholipid vesicles was found to be advantageous for the treatment of skin lesions [22-24].

Among the existing natural antioxidants, mangiferin is less used and studied in modern phytotherapy, despite its wide use in traditional Chinese herbal preparations [25]. Mangiferin is a glucosylxanthone (2- $\beta$-D-glucopyranosyl1,3,6,7-tetrahydroxy-9H-xanthen-9-one) isolated from higher plants, as well as from mango leaves, fruits and by-products (i.e., peel and kernel seed). Research has shown the antioxidant, anti-inflammatory, antidiabetic, anticancer, antimicrobial, analgesic and immunomodulatory properties of mangiferin [26,27]. However, mangiferin has a very low solubility in aqueous media $(0.111 \mathrm{mg} / \mathrm{ml})$ [28] and poor lipophilicity [29], and its efficacy in vivo is hampered by low absorption and high speed of clearance. The bioavailability at skin level is low and, when applied as a cream or gel, it hardly crosses the stratum corneum (SC) and remains on the skin surface [30,31]. For these reasons, the incorporation of mangiferin in phospholipid vesicles represents a valuable approach to increase its bioavailability and absorption at skin level. To this purpose, transfersomes can be effectively employed, since they are phospholipid vesicles specifically designed for dermal and transdermal delivery. The novelty of this work lies in the fact that, differently from what has been proposed previously, we modified transfersomes by adding three key components: glycerol, propylene glycol and mucin to improve the penetration enhancing capabilities, the stability and the mucoadhesive properties $[32,33]$ of the vesicles. Glycerol is a well-known moisturizing agent that increases the hydration of human skin [34], and an osmolyte that stabilizes fluid structures in phospholipid bilayer systems (both skin lamellar pathways and vesicle carriers) [35]. Propylene glycol is a water-soluble, biocompatible penetration enhancer widely used in pharmaceutical preparations; it is able to improve drug permeation through the skin from topical preparations [36]. Mucins are large glycoproteins ubiquitous in the animal kingdom, which can exert barrier, hydration, adhesion and lubrication properties [37]. Glycerol and propylene glycol have been previously used individually to ameliorate the performance of phospholipid vesicles, but, in this work, they were used in combination, either in the presence or absence of mucin, to modify transfersomes for the delivery of mangiferin to the skin. Transfersomes, used as a reference, were prepared by adding Tween 80 to phospholipid vesicles. Tween 80 acts as an edge activator, modifying the bilayer assembly and fluidity. Glycoltransfersomes and mucin-glycoltransfersomes were formulated by combining transfersomes with the glycols and mucin. The vesicles were fully characterized, and their ability to improve the skin delivery of mangiferin and its efficacy in repairing damaged skin were evaluated in vitro and in vivo.

\section{Materials \& methods \\ Materials}

Soy lecithin, glycerol, propylene glycol and Tween 80 were purchased from Galeno Srl (Milan, Italy). Mucin from porcine stomach $(640 \mathrm{kDa})$, mangiferin, HPLC-grade methanol and hydrochloric acid, 3-(4,5-dimethylthiazol-2yl)-3,5 diphenyl tetrazolium bromide (MTT), dimethyl sulfoxide (DMSO), 12-O-tetradecanoylphorbol 13-acetate (TPA) and all the other reagents of analytical grade were purchased from Merck Life Science Srl (Milan, Italy). Cell medium, fetal bovine serum, penicillin, streptomycin and all the other reagents for cell studies were purchased from Thermo Fisher Scientific Inc. (MA, USA).

\section{Vesicle preparation}

Glycoltransfersomes were prepared in two steps. In the first step, mangiferin $(20 \mathrm{mg})$ was weighed in a glass vial and dispersed in a hydrating blend $(2 \mathrm{ml})$ of water, glycerol and propylene glycol $(25: 25: 50, \% \mathrm{v} / \mathrm{v})$ and sonicated ( 25 cycles, $5 \mathrm{~s}$ on and $2 \mathrm{~s}$ off, 13 microns of probe amplitude) by using a high intensity ultrasonic disintegrator (Soniprep 150, MSE Crowley, London, UK). In the second step, $360 \mathrm{mg}$ of phospholipid and $40 \mathrm{mg}$ of Tween 80 were weighed in a glass vial, the mangiferin in the water, glycerol and propylene glycol blend was added, and the final dispersion was sonicated ( 25 cycles, $5 \mathrm{~s}$ on and $2 \mathrm{~s}$ off, 13 microns of probe amplitude). The composition of samples is reported in Supplementary Table 1. 
Transfersomes (used as a reference) were prepared by dispersing the phospholipid (360 mg), Tween 80 (40 mg) and mangiferin $(20 \mathrm{mg})$ in water $(2 \mathrm{ml})$, and sonicating the dispersion $(25$ cycles, $5 \mathrm{~s}$ on and $2 \mathrm{~s}$ off, 13 microns of probe amplitude) [38]. In addition, muc-glycoltransfersomes and muc-transfersomes were prepared by adding the polymer (mucin, $2 \mathrm{mg} / \mathrm{ml}$ ) to the disperions.

Each vesicle dispersion (2 ml) was loaded into Spectra/Por ${ }^{\circledR}$ membranes (12-14 kDa MW cutoff, 3-nm pore size; Spectrum Laboratories Inc., DG Breda, The Netherlands) and dialysed against water (2 l) for $2 \mathrm{~h}$ (with water refreshed after $1 \mathrm{~h}$ ) at room temperature, to remove the nonincorporated mangiferin [39].

\section{Vesicle characterization}

Cryo-TEM analyses were performed to evaluate vesicle formation and morphology. Each vesicle dispersion, properly diluted with water, was placed on a glow-discharged holey carbon grid and vitrified with ethane by using a Vitrobot System (FEI Company, Eindhoven, The Netherlands). The vitreous film was observed under a transmission electron microscopy (TEM) microscope (Tecnai F20, FEI Company) in a low-dose mode at $200 \mathrm{kV}$ and at approximately $-173^{\circ} \mathrm{C}$.

The average diameter and polydispersity index (PI) (i.e., a measure of the size distribution width) of the vesicles were determined by Dynamic Light Scattering using a Zetasizer nano-ZS (Malvern Instruments, Worcestershire, UK), which uses a helium-neon laser $(633 \mathrm{~nm})$ with a $173^{\circ}$ scattering angle and backscatter technology for high sensitivity. The $\zeta$ potential (ZP) was estimated by electrophoretic light scattering using the Zetasizer nano-ZS, which measures the electrophoretic mobility of particles in dispersion. The measurements were carried out at $25^{\circ} \mathrm{C}$ after a 1:100 dilution of the samples with the dispersing medium used for their preparation (water or water, glycerol and propylene glycol blend) [40].

The stability of the vesicles was assessed by monitoring the mean diameter (MD), PI and ZP over 6 months at $25 \pm 1^{\circ} \mathrm{C}$.

Entrapment efficiency was expressed as the percentage of mangiferin recovered after dialysis versus the amount found in nondialysed samples. Mangiferin was quantified by HPLC after disruption of nondialysed and dialysed vesicles with methanol (1:100 dilution), using a Perkin Elmer Flexar chromatograph (Madrid, Spain) equipped with a UV detector and a column Brisa LC2 C18 $(5 \mu \mathrm{m}, 15 \times 0.46 \mathrm{~cm}$; Teknokroma, Barcelona, Spain). The isocratic mobile phase consisted of a mixture of hydrochloric acid $(\mathrm{pH} 4.0)$ and methanol $(60: 40, \mathrm{v} / \mathrm{v})$, the flow rate was $1.2 \mathrm{ml} / \mathrm{min}$, and the injection volume was $20 \mu \mathrm{l}$. The detection wavelength was set at $254 \mathrm{~nm}$. Standard calibration curves covering the whole mangiferin range concentrations were built. The obtained calibration graphs showed a good linearity $\left(\mathrm{R}^{2}=0.99\right)$. The limits of detection and quantification were estimated using the calibration curve procedure and were 0.21 and $0.54 \mu \mathrm{g} / \mathrm{ml}$, respectively. Accuracy was evaluated in terms of error relative and precision by means of coefficient of variation, both values were less than $7 \%$.

\section{Evaluation of skin delivery performances of mangiferin-loaded vesicles}

Experiments were performed by using newborn pig skin provided by a local slaughterhouse. The skin was stored at $-80^{\circ} \mathrm{C}$ after excision, defrosted and pre-equilibrated at $37^{\circ} \mathrm{C}$ in saline $2 \mathrm{~h}$ before the experiments. The fullthickness skin specimens ( $\mathrm{n}=6$ per formulation) were sandwiched between the donor and receptor compartments of Franz vertical cells with an effective diffusion area of $0.784 \mathrm{~cm}^{2}$. The receptor compartment was filled with an aqueous solution of Tween $80(1 \% ; \sim 6 \mathrm{ml})$, which was continuously stirred with a small magnetic bar and thermostated at $37 \pm 1^{\circ} \mathrm{C}$ throughout the experiment to reach the physiological temperature (i.e., $32 \pm 1^{\circ} \mathrm{C}$ ) of the skin surface [41]. The mangiferin formulations were applied $(200 \mu \mathrm{l})$ onto the skin surface, under nonocclusive conditions. A dispersion of mangiferin in a water, glycerol and propylene glycol blend (25:25:50, \%v/v) was used as a reference. Every $2 \mathrm{~h}$ and up to $24 \mathrm{~h}$, the receiving solution was withdrawn, replaced with an equal volume of a fresh solution, and analyzed by HPLC for mangiferin content (see 'Vesicle characterization' section). At the end of the experiment, the skin surface was gently washed with water and blotted dry on absorbent paper. SC was stripped with adhesive tape (Tesa ${ }^{\circledR}$ AG, Hamburg, Germany) and epidermis was separated from dermis with a scalpel. The tape strips, epidermis and dermis were cut, placed separately in vials containing methanol and sonicated in an ice bath $(2 \mathrm{~min})$ to extract mangiferin. After filtration, the solutions were assayed for mangiferin content by HPLC (see 'Vesicle characterization' section) [21]. 
Evaluation of biocompatibility \& protective effect of mangiferin-loaded vesicles against oxidative damage in vitro

Mouse embryonic fibroblasts (3T3) (ATCC collection, VA, USA) were grown as monolayers in $75-\mathrm{cm}^{2}$ flasks incubated with $100 \%$ humidity and $5 \% \mathrm{CO}_{2}$ at $37^{\circ} \mathrm{C}$. DMEM with high glucose, $10 \%$ fetal bovine serum, $1 \%$ penicillin or streptomycin and fungizone, was used to culture the cells. Cell viability was measured by the 3-(4,5-dimethylthiazol-2-yl)-3,5 diphenyl tetrazolium bromide (MTT) test [42]. The cells $\left(7.5 \times 10^{3} /\right.$ well) were seeded into 96-well plates, cultured for $24 \mathrm{~h}$ and exposed for $48 \mathrm{~h}$ to the samples (i.e., mangiferin in a water, glycerol and propylene glycol dispersion, or loaded in vesicles) properly diluted with medium to achieve the desired concentration of mangiferin $(10,1,0.1$ and $0.01 \mu \mathrm{g} / \mathrm{ml})$. Thereafter, an MTT solution $(0.5 \mathrm{mg} / \mathrm{ml}$ final concentration) was added to each well, removed after $3 \mathrm{~h}$ and replaced with DMSO, then the absorbance of the solubilized dye was read at $570 \mathrm{~nm}$ with a microplate reader (Multiskan EX, Thermo Fisher Scientific Inc.). The results are shown as a percentage of live cells in comparison with untreated control cells ( $100 \%$ cell viability).

To evaluate the protective ability of the vesicles against oxidative damage, the cells were seeded into 96-well plates, incubated for $24 \mathrm{~h}$ and exposed simultaneously to hydrogen peroxide (1:50,000 dilution) and mangiferin in a water, glycerol and propylene glycol dispersion or loaded in vesicles $(1 \mu \mathrm{g} / \mathrm{ml}$ mangiferin). After $4 \mathrm{~h}$, the cells were washed with phosphate-buffered solution (PBS), and the MTT assay was performed to evaluate the protective effect of the mangiferin samples against death caused by oxidative stress [21]. Untreated cells (100\% viability) were used as a negative control, and cells exposed to hydrogen peroxide only were used as a positive control.

\section{Scratch wound healing assay}

The wound healing properties of the vesicle formulations was assessed by measuring the expansion of fibroblasts on the wound surface. 3T3 cells were grown in six-well plates to form a confluent monolayer, and a mechanical wound was created by scratching with a sterile plastic pipette tip. Cell fragments were gently washed out with fresh medium. The cells were incubated with the vesicle formulations for $48 \mathrm{~h}$. Untreated cells were used as a control, as well as cells treated with a dispersion of mangiferin in a water, glycerol and propylene glycol blend. The rate of cell migration leading to wound closure was observed under a light microscope with a $10 \times$ objective, capturing images at scheduled intervals, thus assessing the efficacy of the vesicle formulations in wound healing.

\section{Evaluation of protective effect of mangiferin-loaded vesicles against skin damage in vivo}

For the inflammation study, female CD-1 mice (5-6 weeks old, 25-35 g) were supplied by Envigo laboratories (Barcelona, Spain). Mice were acclimatized for 1 week before use. The experiments were performed in accordance with the European regulations for handling and use of laboratory animals, and the protocols were approved by the Institutional Animal Care and Use Committee of the University of Valencia (code 2018/VSC/PEA/0032 type 2). The mice $(\mathrm{n}=4)$ were divided in groups, including untreated animals (negative control), animals treated with TPA and saline (positive control), and animals treated with the vesicle formulations or a dispersion of mangiferin in a water, glycerol and propylene glycol blend. The back skin of mice was shaved 1 day before the experiment. On day 1 , cutaneous inflammation and lesion were induced by applying 12-O-tetradecanoylphorbol 13-acetate (TPA; $20 \mu \mathrm{l})$ dissolved in acetone $(243 \mu \mathrm{M})$ on the shaved area $\left(\sim 2 \mathrm{~cm}^{2}\right)$. After $3 \mathrm{~h}, 200 \mu \mathrm{l}$ of each sample was topically smeared over the TPA-treated area until complete absorption. The procedure was repeated on day 2 and 3 . On day 4 , mice were sacrificed by cervical dislocation, and the treated skin area was excised and immediately stored at $-80^{\circ} \mathrm{C}$.

The myeloperoxidase (MPO) activity, a quantitative assessment of neutrophil infiltration into the skin, was measured [40]. Mouse skin biopsies were homogenized and centrifuged, the supernatant $(10 \mu \mathrm{l})$ was incubated with sodium phosphate buffer $(\mathrm{pH} 5.4 ; 20 \mu \mathrm{l})$, phosphate buffer $(\mathrm{pH} 7.4 ; 200 \mu \mathrm{l}), 0.052 \%$ hydrogen peroxide $(40 \mu \mathrm{l})$

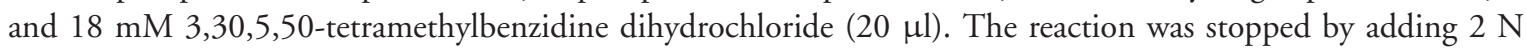
$\mathrm{H}_{2} \mathrm{SO}_{4}(50 \mu \mathrm{l})$. The absorbance was read at $450 \mathrm{~nm}$. The MPO activity was calculated from the linear portion of a standard curve and expressed as $\mathrm{ng} / \mathrm{ml}$.

\section{Histological examination}

Skin biopsies (see 'Evaluation of protective effect of mangiferin-loaded vesicles against skin damage in vivo' section) were excised from the treated mice dorsal region, after $72 \mathrm{~h}$ of treatment (on day 4) and maintained in formaldehyde $(10 \% \mathrm{v} / \mathrm{v})$ for microscopic studies. Tissue samples were processed routinely and embedded in paraffin wax. 

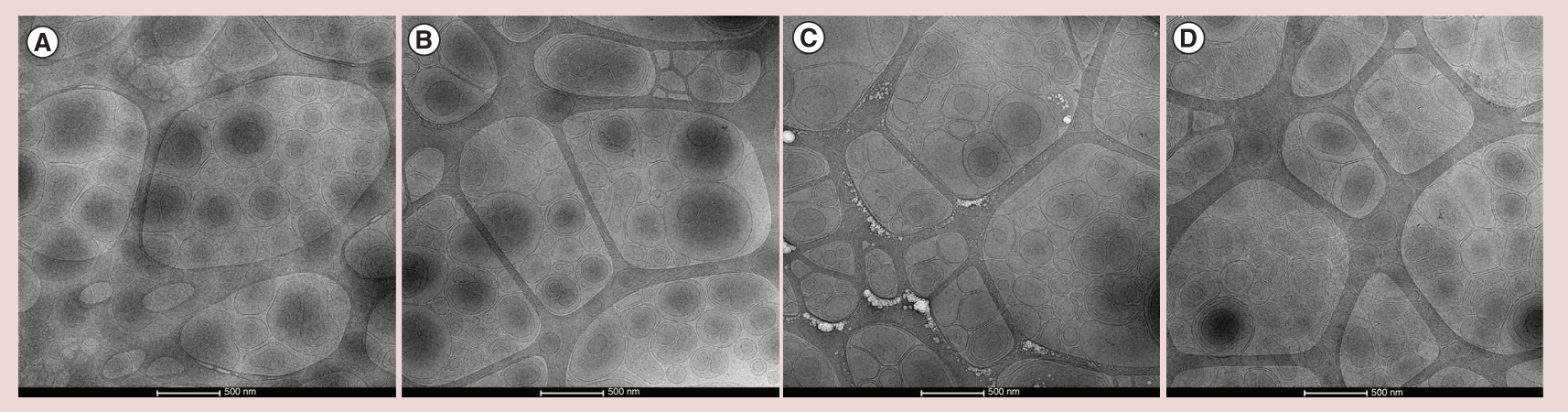

Figure 1. Cryo-transmission electron microscopy images. Representative images of mangiferin-loaded vesicles: transfersomes (A), glycoltransfersomes (B), muc-transfersomes (C), muc-glycoltransfersomes (D).

Longitudinal sections $(5 \mu \mathrm{m})$ were stained with hematoxylin and eosin. Microscopic assessment by light microscope was performed blind on coded slices.

\section{Statistical analysis of data}

Results are expressed as means \pm standard deviations. Analysis of variance was used to evaluate multiple comparison of means and Tukey's test and Student's t-test were performed to substantiate differences between groups using XLStatistics for Windows. The differences were considered statistically significant for $\mathrm{p}<0.05$.

\section{Results}

Vesicle preparation \& characterization

A pre-formulation study was carried out using different types and amounts of phospholipid, edge activators and water cosolvents. Among the numerous formulations tested, the vesicles made of soy lecithin $(180 \mathrm{mg} / \mathrm{ml})$, mangiferin $(10 \mathrm{mg} / \mathrm{ml})$ and Tween $80(20 \mathrm{mg} / \mathrm{ml})$ in a blend of water, glycerol and propylene glycol (25:25:50\% $\mathrm{v} / \mathrm{v}$ ) were selected based on their small size and stability. Given their composition, the vesicles were named glycoltransfersomes. Transfersomes with the same concentration of soy lecithin, mangiferin and Tween 80 were prepared as a reference. Furthermore, transfersomes and glycoltransfersomes were modified by the addition of mucin, which is expected to ameliorate the adhesion of the vesicles to skin and cells [43,44].

Cryo-TEM observation confirmed the formation of lamellar vesicles (Figure 1). In particular, in transfersomes (Figure 1A), unilamellar, fairly spherical, small vesicles were observed; in glycoltransfersomes and muc-transfersomes (Figure 1B \& C), uni- and oligolamellar vesicles coexisted with multivesicular structures; in muc-glycoltransfersomes (Figure 1D), multilamellar, larger and more irregularly shaped vesicles were evident. These results suggest that the addition of glycols and mucin impact the morphology of the vesicles.

The MD, PI, ZP and entrapment efficiency of the vesicles were first evaluated (Table 1). Empty vesicles (i.e., without mangiferin) were prepared and characterized to evaluate the effect of the payload on the vesicle assembly.

Transfersomes were the smallest vesicles $(\sim 75 \mathrm{~nm}$; Table 1$)$ and their size was not affected by the presence of mangiferin or mangiferin and mucin together $(p>0.05$ among empty transfersomes, mangiferin transfersomes and mangiferin muc-transfersomes). The addition of mucin, without mangiferin, caused an increase in the MD of muc-transfersomes ( $\sim 150 \mathrm{~nm}, \mathrm{p}<0.05$ vs the other transfersomes; Table 1). Empty glycoltransfersomes and empty muc-glycoltransfersomes showed the largest MD (292 and $238 \mathrm{~nm}$, respectively). Both systems underwent a decrease in size upon addition of mangiferin: the decrease was strong in the case of glycoltransfersomes $(\sim 100 \mathrm{~nm})$ and much less marked in the case of muc-glycoltransfersomes $(\sim 210 \mathrm{~nm})$. The vesicle enlargement seems to be dependent on the addition of the glycols and mucin, and mangiferin can mitigate these modifications, even though mangiferin-loaded muc-glycoltransfersomes still display a large size. We can hypothesize that the glycols and mucin interact with the bilayer surface decreasing the repulsion between concentric bilayer and leading to the formation of multilamellar, large vesicles (as confirmed by cryo-TEM). 


\begin{tabular}{|c|c|c|c|c|}
\hline Sample & $\begin{array}{l}\text { MD } \\
(\mathrm{nm})\end{array}$ & PI & $\begin{array}{l}\mathrm{ZP} \\
(\mathrm{mV})\end{array}$ & $\begin{array}{l}\mathrm{EE} \\
(\%)\end{array}$ \\
\hline Empty transfersomes & $77 \pm 9$ & 0.22 & $-39 \pm 7$ & - \\
\hline Empty muc-transfersomes & $146 \pm 32$ & 0.24 & $-42 \pm 6$ & - \\
\hline Empty glycoltransfersomes & $292 \pm 41$ & 0.26 & $-24 \pm 8$ & - \\
\hline Empty muc-glycoltransfersomes & $238 \pm 11$ & 0.26 & $-60 \pm 5$ & - \\
\hline Mangiferin transfersomes & $73 \pm 5$ & 0.22 & $-56 \pm 4$ & $91 \pm 5$ \\
\hline Mangiferin muc-transfersomes & $78 \pm 5$ & 0.26 & $-43 \pm 3$ & $94 \pm 7$ \\
\hline Mangiferin glycoltransfersomes & $98 \pm 33$ & 0.16 & $-72 \pm 4$ & $92 \pm 3$ \\
\hline Mangiferin muc-glycoltransfersomes & $212 \pm 15$ & 0.25 & $-61 \pm 5$ & $91 \pm 4$ \\
\hline
\end{tabular}

The PI of all the formulations was $\leq 0.26$, confirming the formation of monodispersed systems. The $\mathrm{ZP}$ was always highly negative, especially for mangiferin-loaded glycoltransfersomes $(>-60 \mathrm{mV})$. All the vesicles were able to load mangiferin in good yields, as indicated by the entrapment efficiency values ( $>92 \%)$, without significant differences among the formulations $(\mathrm{p}>0.05)$.

In order to evaluate the stability of the vesicles during storage, the dispersions were stored at $25^{\circ} \mathrm{C}$ in the dark for 6 months, and their size, PI and ZP were measured at scheduled times (Figure 2). The study showed a good stability: the MD of transfersomes and muc-transfersomes increased up to approximately $115 \mathrm{~nm}$ already after 3 months, while that of glycoltransfersomes and muc-glycoltransfersomes remained constant, which points to a positive contribution of glycols to vesicle stability. In particular, results underlined that mucin did not interfere with the transferosomes assembly, while the combination of glycols and mucin led to a significant increase in vesicle size, even though the effect of the polymer on vesicle stability was negligible as the tested parameters remained unchanged over 6 months of storage. The PI remained almost constant for all the formulations, and the ZP slightly decreased, still being highly negative (Figure 2 A \& B).

\section{Evaluation of skin delivery performances of vesicles}

The ability of transfersomes and glycoltransfersomes to enhance the deposition of mangiferin into the skin strata was evaluated by using Franz diffusion cells and newborn pig skin. The deposited mangiferin was extracted from the skin and quantified by HPLC (Figure 3).

When mangiferin was applied in a water-glycol blend, the highest amount was found in the SC ( $4 \%)$, while in the deeper strata the overall amount was $>0.5 \%$. Lower values were found using transfersomes: the highest deposition was in the SC, but lower than that provided by the dispersion $(\sim 2 \%, \mathrm{p}<0.05)$, with a greater accumulation in the dermis $(\sim 0.5 \%)$. This points to a contribution of the glycols in facilitating the diffusion of mangiferin toward the deep dermis. The addition of mucin to transfersomes (muc-transfersomes) led to a further decrease of mangiferin deposition in the SC $(\sim 0.6 \%, \mathrm{p}<0.05$ vs dispersion and transfersomes) and an increase in the epidermis ( $\sim 1.1 \%, \mathrm{p}<0.05$ vs dispersion and transfersomes). The best results were obtained when mangiferin was loaded in glycoltransfersomes and muc-glycoltransfersomes, which provided similar values $(p>0.05)$. The deposition of mangiferin was especially increased in the epidermis $(\sim 5.3 \%, \mathrm{p}<0.05$ vs dispersion, transfersomes and muc-transfersomes) using both glycoltransfersomes and muc-glycoltransfersomes. In the SC and dermis, the deposition was approximately $1 \%$ using glycoltransfersomes, and $1.5 \%$ using muc-glycoltransfersomes. Hence, the accumulation of mangiferin provided by the glycol-vesicles in epidermis and dermis was approximately fiveto six-fold higher than that obtained using muc-transfersomes, probably due to a synergistic action of glycols and phospholipid vesicles.

\section{Evaluation of the biocompatibility of vesicles}

Fibroblasts, the main cells of human dermis, were incubated with mangiferin-loaded vesicles to evaluate their biocompatibility. The mangiferin dispersed in a water-glycol blend was used as a reference. The vesicles were used at different dilutions corresponding to $10,1,0.1$ and $0.01 \mu \mathrm{g} / \mathrm{ml}$ of mangiferin. When mangiferin in dispersion was tested at lower concentrations $(1,0.1$ and $0.01 \mu \mathrm{g} / \mathrm{ml})$, the viability of fibroblasts was approximately $130 \%$, 

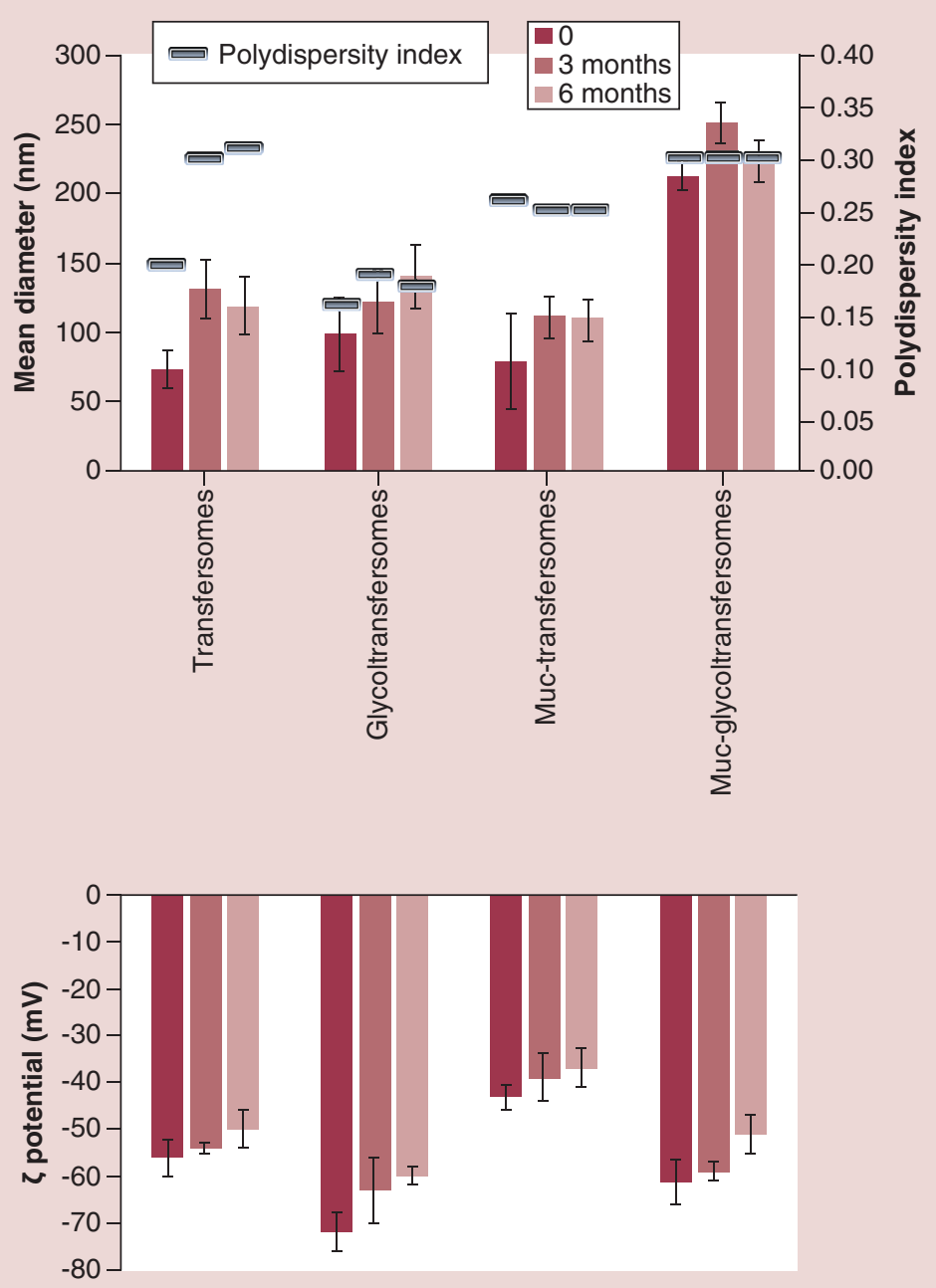

Figure 2. Mean diameter, polydispersity index and $\zeta$ potential of vesicles measured during 6 months of storage at $25^{\circ} \mathrm{C}$. The mean values \pm standard deviations (error bars) are reported $(n=6)$.

which indicates a proliferative effect of the polyphenol, while when the higher concentration $(10 \mu \mathrm{g} / \mathrm{ml} \mathrm{of}$ mangiferin) was tested, the viability was approximately $80 \%$, which indicates a slight toxicity of mangiferin at such concentration. When the mangiferin-loaded transfersomes and muc-transfersomes were used, the behavior was similar: the viability was approximately $95 \%$ using the higher concentration $(10 \mu \mathrm{g} / \mathrm{ml})$ and around $150 \%$ when the lower concentrations $(1,0.1$ and $0.01 \mu \mathrm{g} / \mathrm{ml})$ were used. When the glycoltransfersomes were applied, cell viability was always $>130 \%$, irrespective of the concentration (Figure 4, upper panel). The presence of mucin in muc-glycoltransfersomes slightly reduced the proliferative effect of mangiferin at the higher concentrations (10 and $1 \mu \mathrm{g} / \mathrm{ml}$ ), even though the viability was still around $100 \%$.

\section{Evaluation of the protective effect of the formulations against cell damage caused by oxidative stress}

Given the well-known antioxidant and protective properties of mangiferin, fibroblasts were stressed with hydrogen peroxide, a strong oxidizing agent, and treated with mangiferin-loaded vesicles to evaluate their ability to counteract oxidative damage and consequent cell death. A water-glycol dispersion of mangiferin was used as a reference to estimate the advantages provided by the incorporation in vesicles (Figure 4, lower panel).

After $4 \mathrm{~h}$ of incubation, hydrogen peroxide caused an approximate $60 \%$ mortality of fibroblasts ( $40 \%$ viability). The treatment of stressed fibroblasts with the mangiferin dispersion led to a approximately $20 \%$ reduction of 


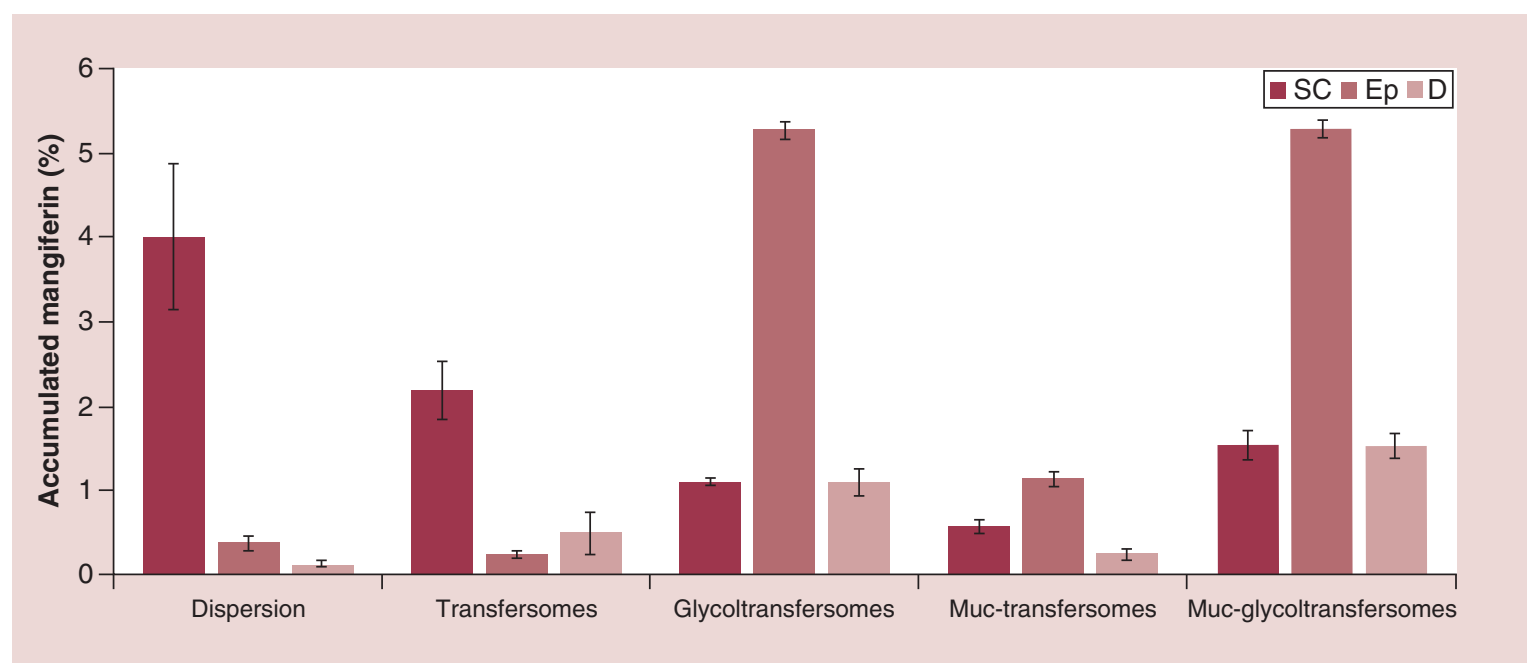

Figure 3. Percentage of mangiferin accumulated in stratum corneum, epidermis and dermis after $24 \mathrm{~h}$ of application of mangiferin in dispersion or loaded into transfersomes, glycoltransfersomes, muc-transfersomes or muc-glycoltransfersomes. Mean values \pm standard deviations are reported $(n=6)$.

D: Dermis; Ep: Epidermis; SC: Stratum corneum.

mortality ( $\sim 60 \%$ viability), and the treatment with mangiferin-loaded vesicles prevented cell mortality completely, as the viability was around $100 \%$, regardless of the formulation tested ( $\mathrm{p}>0.05$ among samples).

\section{Scratch wound healing assay}

The ability of the mangiferin formulations to promote the migration and proliferation of fibroblasts was assessed by producing a linear cut on the cell monolayer and evaluating the closure of the wound during $48 \mathrm{~h}$ (Figure 5).

The closure of the lesion in untreated cells was not complete after $48 \mathrm{~h}$. The treatment of the wound with mangiferin in dispersion stimulated cell proliferation and migration, but at $48 \mathrm{~h}$ the scratch was not completely closed and some areas without cells were still present. A similar behavior was observed using mangiferin-loaded transfersomes. On the other hand, when mangiferin-loaded glycoltransfersomes, muc-transfersomes and mucglycoltransfersomes were used, the closure of the wound was complete already at $32 \mathrm{~h}$.

\section{Evaluation of the protective effect of mangiferin formulations against skin damage}

Given the need to reduce the number of animals for ethical issues, only the most promising vesicle formulations were tested in vivo: mangiferin-loaded glycoltransfersomes, muc-transfersomes and muc-glycoltransfersomes. The skin of the mice injured by TPA and treated with the mangiferin dispersion appeared severely damaged, similarly to the skin treated with saline (positive control): it appeared desquamated and covered with necrotic tissue (Figure 6).

The skin of mice treated with mangiferin-loaded muc-transfersomes was almost completely healed, but still showing some minor lesions. On the other hand, when mangiferin-loaded glycoltransfersomes and mucglycoltransfersomes were applied, the skin appeared undamaged by TPA and comparable with untreated skin (negative control).

The activity of MPO, an enzyme released by neutrophils and macrophages during inflammatory processes, was measured to evaluate the degree of skin inflammation. The MPO concentration in the tissue inflamed by TPA application and treated with saline was approximately $50 \mathrm{ng} / \mathrm{ml}$. The treatment with mangiferin dispersion allowed a decrease of this value to approximately $34 \mathrm{ng} / \mathrm{ml}(\mathrm{p}<0.05)$, and the treatment with mangiferin-loaded muc-transfersomes led to a further decrease $(\sim 25 \mathrm{ng} / \mathrm{ml}, \mathrm{p}<0.05$ vs mangiferin dispersion). The most significant reduction of MPO was observed when mangiferin-loaded glycoltransfersomes and muc-glycoltransfersomes were used, reaching values approximately 3.3 -fold lower than that of skin treated with saline $(\sim 15 \mathrm{ng} / \mathrm{ml}, \mathrm{p}<0.05$ vs the other formulations). 

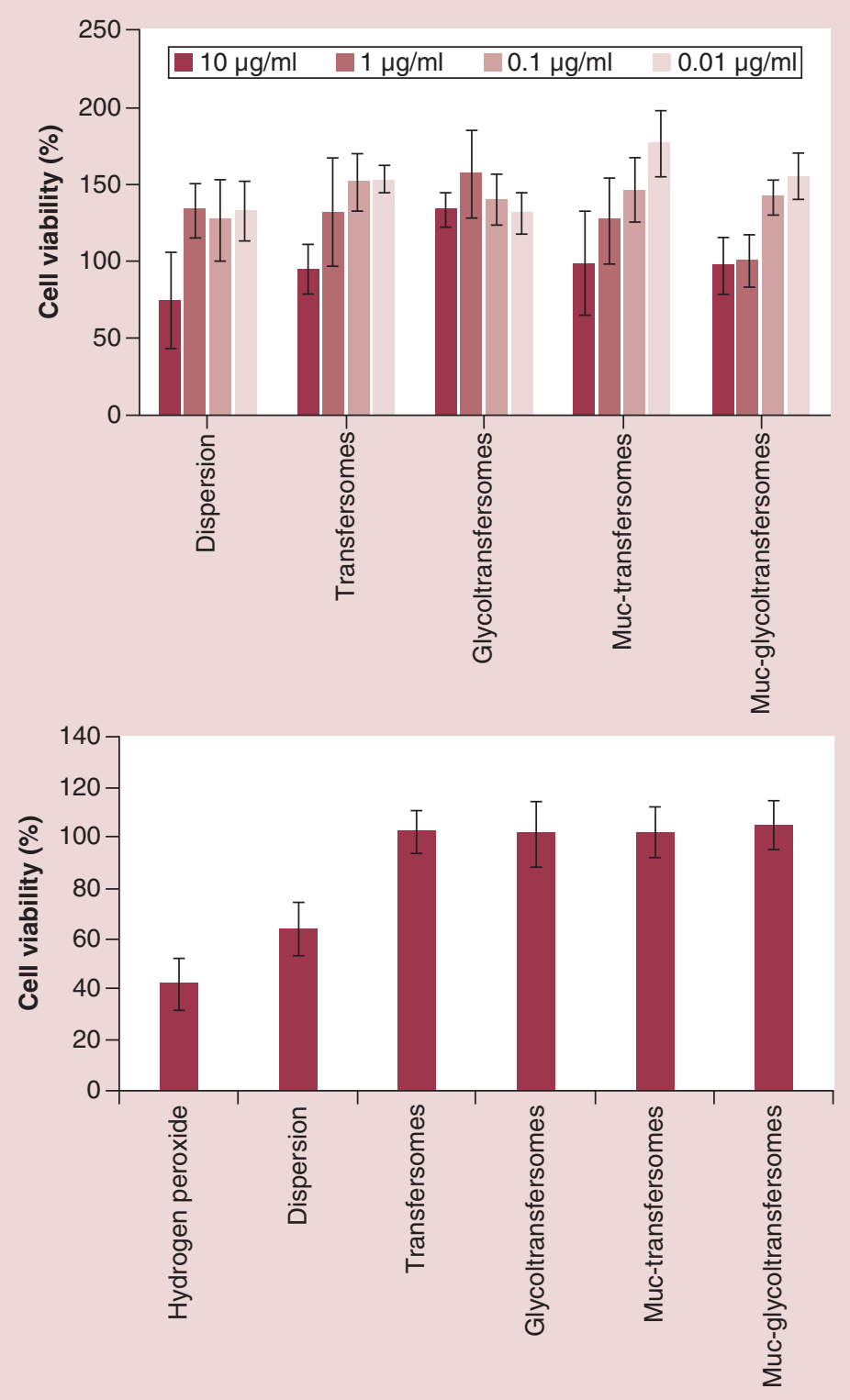

Figure 4. Viability values for fibroblasts. Viability values for fibroblasts treated for $48 \mathrm{~h}$ with mangiferin in dispersion or loaded in vesicles (upper panel). Viability values for fibroblasts stressed for $4 \mathrm{~h}$ with hydrogen peroxide without treatment or stressed with hydrogen peroxide and treated with mangiferin in dispersion or loaded in vesicles (lower panel). Mean values \pm standard deviations (error bars) are reported $(n=12)$.

\section{Histological examination}

The skin tissues excised from mice treated with TPA and the mangiferin formulations were stained with hematoxylin-eosin and observed under an optical microscope to evaluate the degree of inflammation and ulceration. The skin injured by TPA and treated with saline was characterized by acute inflammation in the dermis (disordered cell organization), skin ulceration and severe panniculitis (Figure 7B). In the skin treated with the mangiferin dispersion (Figure $7 \mathrm{C}$ ), cutaneous ulceration, severe inflammatory infiltration in the dermis and moderate panniculitis were evident. The skin treated with mangiferin-loaded muc-transfersomes (Figure 7E) appeared healthy, but inflammatory infiltration was evident at the dermal level. In the skin treated with mangiferin-loaded glycoltransfersomes (Figure 7D) and muc-glycoltransfersomes (Figure 7F), epidermis did not show any lesions and the inflammatory infiltration at dermal level was moderate. 


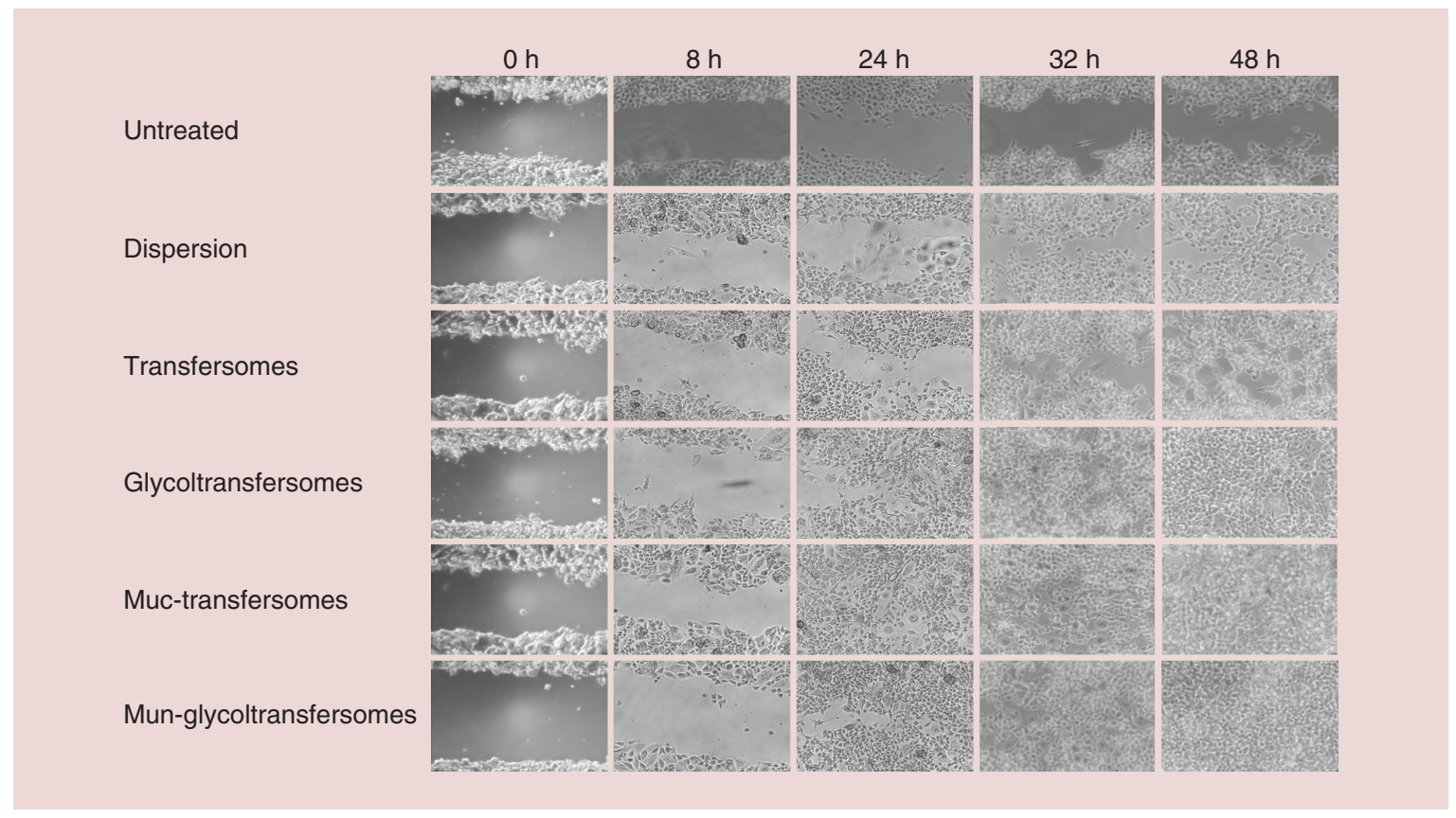

Figure 5. Representative optical microscopy images of wound closure in fibroblasts treated with mangiferin in dispersion or loaded in vesicles during $48 \mathrm{~h}$.
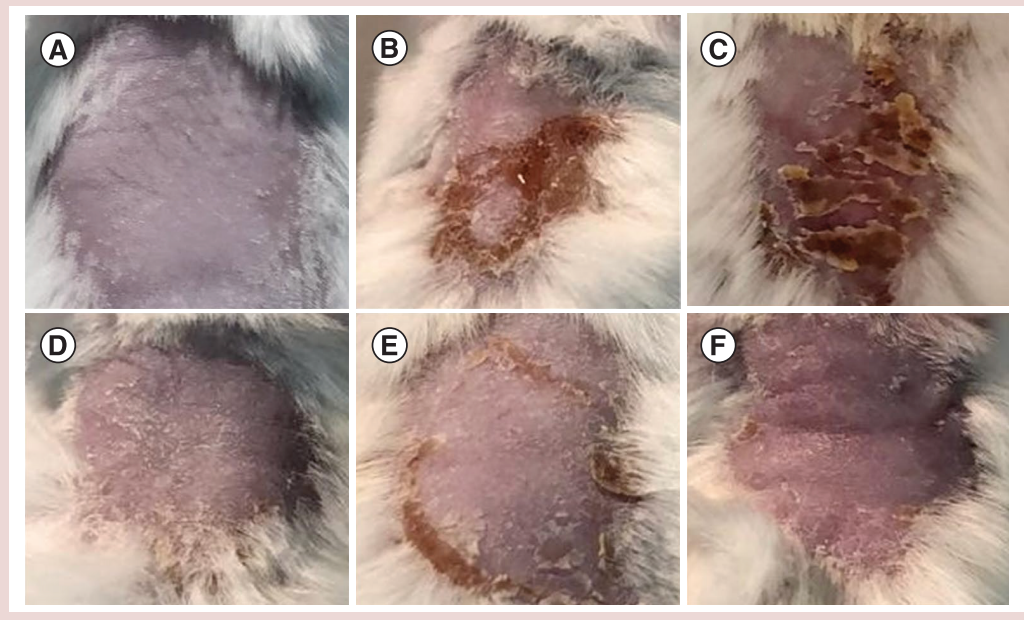

Figure 6. Images of mouse dorsal skin. Representative images of mouse skin: untreated (A), damaged by TPA and treated with saline (B) or treated with mangiferin in dispersion (C), mangiferin loaded in glycoltransfersomes (D), muc-transfersomes (E) and muc-glycoltransfersomes (F).

Hence, the histological results confirmed the superior ability of glycoltransfersomes and muc-glycoltransfersomes to deliver mangiferin to the epidermis and dermis and promote skin regeneration.

\section{Discussion}

During recent years, the development of nanosystems for the delivery of natural antioxidants has attracted an extensive attention due to promising beneficial effects of the resulting nanophytoformulations [45]. Given that, in this study, mangiferin was loaded in innovative phospholipid vesicles tailored for skin delivery. The most suitable phospholipid vesicles capable of loading $10 \mathrm{mg} / \mathrm{ml}$ of mangiferin were selected based on a preformulation screening. Different types and amounts of phospholipids, edge activators and cosolvents were explored. Soy 


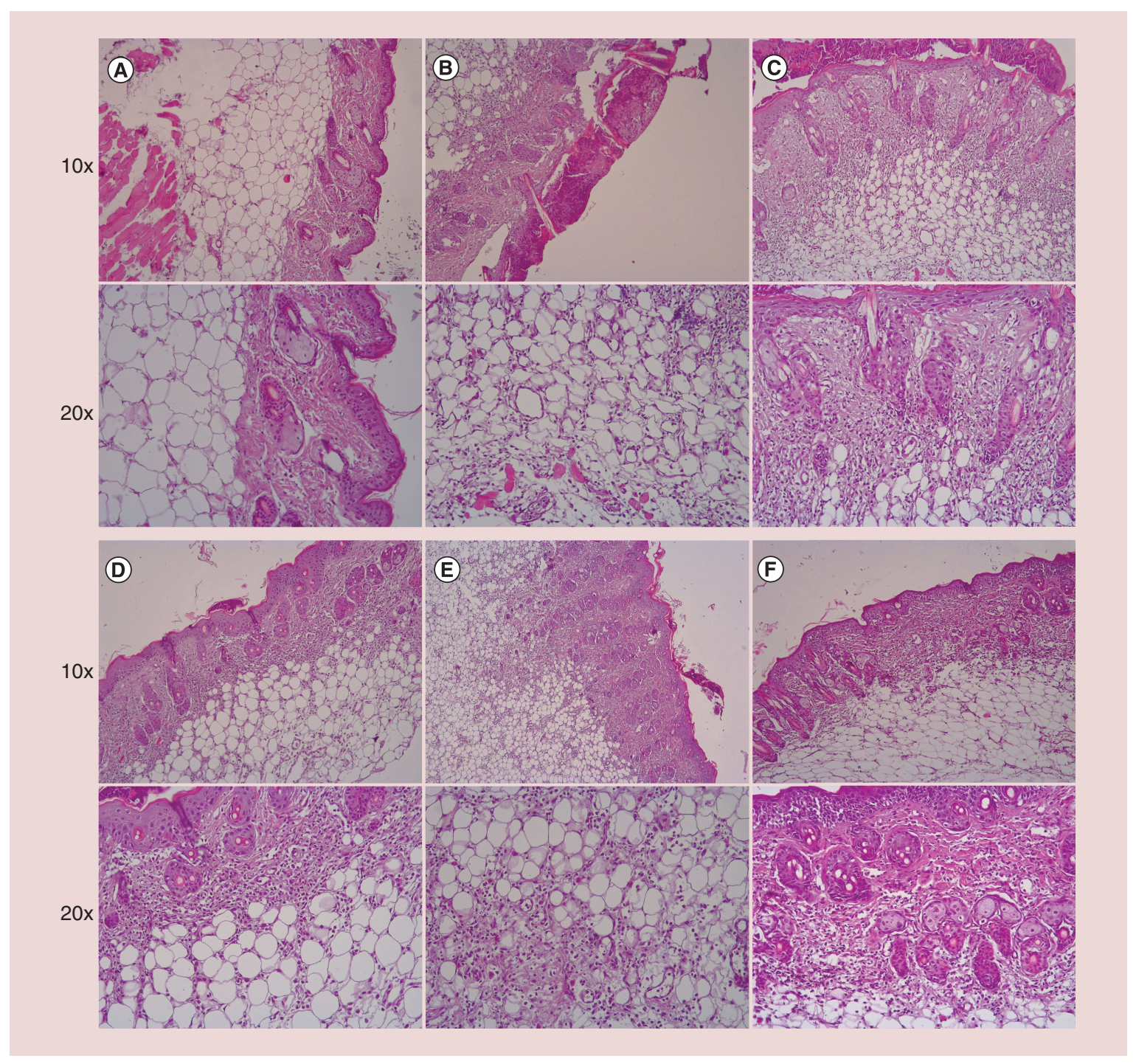

Figure 7. Representative histological images. Representative histological images of skin sections stained with hematoxylin-eosin and observed under an optical microscope with magnification 10× (upper panel) and 20× (lower panel). Untreated skin (A), skin injured by TPA and treated with saline (B) or mangiferin in dispersion (C), mangiferin loaded in glycoltransfersomes (D), muc-transfersomes (E) and muc-glycoltransfersomes (F).

lecithin $(180 \mathrm{mg} / \mathrm{ml})$ was selected as phospholipid, in association with Tween $80(20 \mathrm{mg} / \mathrm{ml})$, which acts as edge activator making the bilayer more fluid [46,47]. The mixture of phospholipid, Tween 80 and mangiferin was hydrated with a water, glycerol and propylene glycol blend $(25: 25: 50, \% \mathrm{v} / \mathrm{v})$, which resulted in the production of glycoltransfersomes characterized by small size and stability over time. We can speculate that propylene glycol acts as a penetration enhancer promoting the skin delivery capabilities of the vesicles [48], and glycerol exerts moisturizing and hydrating effects on the skin [49].

Transfersomes, prepared with the same amount of soy lecithin and Tween 80 and hydrated with water, were used as a reference. Mucin was added to both the transfersomes and glycoltransfersomes to improve the bioadhesive properties and prolong the residence time in the skin [50].

The glycoltransfersomes were around $100 \mathrm{~nm}$ and muc-glycoltransfersomes were around $200 \mathrm{~nm}$. Despite being larger than transfersomes and muc-transfersomes, glycoltransfersomes and muc-glycoltransfersomes were more stable, as the size did not change during the storage period, reasonably due to the presence of the glycols and the multilayered structure of these vesicles. 
The loading of mangiferin in the vesicles induced a reduction of MD and ZP, which indicates an involvement of the polyphenol in the bilayer assembly. Indeed, the mangiferin is a lipophilic molecule that intercalates in the bilayer between the phospholipid chains [51]. Thanks to their favorable composition, glycoltransfersomes and muc-glycoltransfersomes enhanced the delivery of mangiferin to epidermis and dermis, which are the main tissues involved in the repair process of skin lesions [52]. On the contrary, mangiferin dispersion and transfersomes favored the accumulation of the polyphenol in the SC. The total amount of mangiferin deposited in the whole skin after $24 \mathrm{~h}$ of experiment was around $2 \%$ for transfersomes and muc-transfersomes, while using glycoltransfersomes and muc-glycoltransfersomes, the amount was four-times higher $(\sim 8 \%)$, with a predominant accumulation in epidermis and dermis $(\sim 6 \%)$, which was 12-times higher than the amount provided by the aqueous dispersion. This confirms the enhancing properties of the glycol-vesicles. In addition, it is worthy to note that these results refer to intact skin, but our formulations are intended for the treatment of injured skin with impaired barrier function, and hence the delivery of mangiferin is expected to be higher. Indeed, in the in vivo study performed on damaged skin, glycoltransfersomes and muc-glycoltransfersomes, showed superior carrier performances resulting in an improved efficacy of mangiferin.

The vesicles were able to effectively counteract the dangerous effects of oxygen free radicals in dermal fibroblasts and promote their migration and proliferation into the wound site, facilitating its closure. In the in vitro studies in fibroblasts, all the vesicles promoted the efficacy of mangiferin thanks to their carrier capabilities, irrespective of their composition, which instead was a key determinant of the performance of the vesicles in skin delivery, and consequently in the in vivo study. Indeed, the skin treated with mangiferin-loaded glycoltransfersomes and muc-glycoltransfersomes showed a complete remission of the lesions caused by TPA, which was daily applied onto the skin to produce an extended wound associated with edematous and inflamed tissue simulating the biochemical events that naturally occur in in vivo pathological wounds $[53,54]$. The superior performance of glycoltransfersomes can be reasonably ascribed to the glycols, which were not present in transfersomes. It has to be noted that the effect of mucin was negligible. The glycols are supposed to be located both within the vesicles and in the intervesicle medium, and when they come into contact with the skin surface, they promote hydration and perturb the SC barrier, acting synergistically with the phospholipid and the edge activator, thus facilitating the passage of intact vesicles.

\section{Conclusion}

Results disclosed the optimal performances of glycoltransfersomes in delivering mangiferin to damaged skin, potentiating its wound healing properties and confirming the value of the applied formulation strategy. Indeed, the two used water cosolvents, glycerol and propylene glycol, exert a synergistic activity with the elastic vesicles (transfersomes), improving their well-known ability to overcome the biological membranes. These novel carriers maximize the efficacy of mangiferin in tissue regeneration, thus representing promising formulations for wound healing.

\section{Summary points}

- Tween 80, glycerol and propylene glycol were used to formulate glycoltransfersomes.

- Mangiferin, a natural antioxidant, was incorporated in glycoltransfersomes.

- Glycoltransfersomes were modified by adding mucin to increase their performances.

- Both glycoltransfersomes and mucin-glycoltransfersomes promoted the deposition of mangiferin in epidermis and dermis.

- The vesicles were cytocompatible and capable of protecting fibroblasts from oxidative stress.

- The vesicles stimulated fibroblasts' proliferation and migration leading to wound closure in vitro.

- The vesicles protected mouse skin from chemically induced injury.

- The vesicles reduced inflammatory infiltration and promoted skin regeneration in mice.

Supplementary data

To view the supplementary data that accompany this paper please visit the journal website at: www.futuremedicine.com/doi/suppl/10.2217/nnm-2020-0116 
Financial \& competing interests disclosure

This research was financially supported by FSC 2014-2020 - Patto per lo Sviluppo della Regione Sardegna, RASSR14371. The authors have no other relevant affiliations or financial involvement with any organization or entity with a financial interest in or financial conflict with the subject matter or materials discussed in the manuscript apart from those disclosed.

No writing assistance was utilized in the production of this manuscript.

\section{Open access}

This work is licensed under the Attribution-NonCommercial-NoDerivatives 4.0 Unported License. To view a copy of this license, visit http://creativecommons.org/licenses/by-nc-nd/4.0/

\section{References}

Papers of special note have been highlighted as: $\bullet$ of interest; $\bullet \bullet$ of considerable interest

1. Atanasov AG, Waltenberger B, Pferschy-Wenzig EM et al. Discovery and resupply of pharmacologically active plant-derived natural products: a review. Biotechnol. Adv. 33(8), 1582-1614 (2015).

2. Feng T, Wei Y, Lee RJ. Liposomal curcumin and its application in cancer physical property. Int. J. Nanomedicine 12, 6027-6044 (2017).

3. Gokhale JP, Mahajan HS, Surana SJ. Quercetin loaded nanoemulsion-based gel for rheumatoid arthritis: in vivo and in vitro studies. Biomed. Pharmacother. 112, 108622 (2019).

4. Sahibzada MUK, Sadiq A, Sfaidah H et al. Berberine nanoparticles with enhanced in vitro bioavailability: characterization and antimicrobial activity. Drug Des. Devel. Ther. 12, 303-312 (2018).

5. Pandita D, Kumar S, Poonia N, Lather V. Solid lipid nanoparticles enhance oral bioavailability of resveratrol, a natural polyphenol. FRIN 62, 1165-1174 (2014).

6. Castangia I, Caddeo C, Manca ML et al. Delivery of liquorice extract by liposomes and hyalurosomes to protect the skin against oxidative stress injuries. Carbohydr. Polym. 134, 657-663 (2015).

7. Allen TM, Cullis PR. Liposomal drug delivery systems: from concept to clinical applications. Adv. Drug Deliv. Rev. 65(1), 36-48 (2013).

- Uses this paper as reference because it shows the main properties and advantages of liposomes.

8. Sala M, Diab R, Elaissari A, Fessi H. Lipid nanocarriers as skin drug delivery systems: properties, mechanisms of skin interactions and medical applications. Int. J. Pharm. 535(1-2), 1-17 (2018).

- Uses this paper as reference because it showed the the properties and the possible mechanisms of interaction between skin and liposomes.

9. El Maghraby GM, Barry BW, Williams AC. Liposomes and skin: from drug delivery to model membranes. Eur. J. Pharm. Sci. 34(4-5), 203-222 (2008).

10. Cevc G, Gebauer D, Stieber J, Schätzlein A, Blume G. Ultraflexible vesicles, transfersomes, have an extremely low pore penetration resistance and transport therapeutic amounts of insulin across the intact mammalian skin. Biochim. Biophys. Acta 1368(2), 201-215 (1998).

-. Uses this paper as reference because it shows the main properties and advantages of transfersomes.

11. Jain S, Tiwary AK, Sapra B, Jain NK. Formulation and evaluation of ethosomes for transdermal delivery of lamivudine. AAPS PharmSciTech 8(4), E111 (2007).

12. Marto J, Vitor C, Guerreiro A et al. Ethosomes for enhanced skin delivery of griseofulvin. Colloids Surf. B Biointerfaces 146, 616-623 (2016).

13. Touitou E, Dayan N, Bergelson L, Godin B, Eliaz M. Ethosomes - novel vesicular carriers for enhanced delivery: characterization and skin penetration properties. J. Control. Rel. 65(3), 403-418 (2000).

14. Ascenso A, Raposo S, Batista C et al. Development, characterization, and skin delivery studies of related ultradeformable vesicles: transfersomes, ethosomes, and transethosomes. Int. J. Nanomedicine 10, 5837-5851 (2015).

15. Mura S, Manconi M, Sinico C, Valenti D, Maria A. Penetration enhancer-containing vesicles (PEVs) as carriers for cutaneous delivery of minoxidil. Int. J. Pharm. 380(1-2), 72-79 (2009).

16. Manconi M, Sinico C, Caddeo C, Vila AO, Valenti D, Fadda AM. Penetration enhancer containing vesicles as carriers for dermal delivery of tretinoin. Int. J. Pharm. 412(1-2), 37-46 (2011).

- Uses this paper as reference because it shows the advantages connected with the use of penetration enhancers like glycols.

17. Manca ML, Zaru M, Manconi M et al. Glycerosomes: a new tool for effective dermal and transdermal drug delivery. Int. J. Pharm. 455(1-2), 66-74 (2013).

- Uses this paper as reference because it describes for the first time the use of glycerol to modify the assembly and fluidity of liposomes.

18. Odeh F, Al-jaber H, Khater D. Nanoflora - how nanotechnology enhanced the use of active phytochemicals. In: Application of Nanotechnology in Drug Delivery. Sezer AD (Ed.). IntechOpen, London, UK, 343-368 (2014). 
19. Sun D, Zhuang X, Xiang X et al. A novel nanoparticle drug delivery system: the anti-inflammatory activity of curcumin is enhanced when encapsulated in exosomes. YMTHE. 18(9), 1606-1614 (2010).

20. Liu Y, Ma Y, Xu J, Chen Y, Xie J, Yue P. Apolipoproteins adsorption and brain-targeting evaluation of baicalin nanocrystals modified by combination of Tween 80 and TPGS. Colloids Surf. B Biointerfaces 160, 619-627 (2017).

21. Moulaoui K, Caddeo C, Manca ML et al. Identification and nanoentrapment of polyphenolic phytocomplex from Fraxinus angustifolia: in vitro and in vivo wound healing potential. Eur. J. Med. Chem. 89, 179-188 (2015).

22. Caddeo C, Nacher A, Vassallo A et al. Effect of quercetin and resveratrol co-incorporated in liposomes against inflammatory/oxidative response associated with skin cancer. Int. J. Pharm. 513(1-2), 153-163 (2016).

23. Mir-Palomo S, Nácher A, Díez-Sales O et al. Inhibition of skin inflammation by baicalin ultradeformable vesicles. Int. J. Pharm. 511(1), 23-29 (2016).

-. Uses this paper as reference because it shows the potential of transfersomes as topical delivery systems.

24. Pleguezuelos-Villa M, Diez-Sales O, Manca ML et al. Mangiferin glycethosomes as a new potential adjuvant for the treatment of psoriasis. Int. J. Pharm. 573 (2020).

25. Ko HC, Wei BL, Chiou WF. The effect of medicinal plants used in Chinese folk medicine on RANTES secretion by virus-infected human epithelial cells. J. Ethnopharmacol. 107(2), 205-210 (2006).

26. Imran M, Arshad MS, Butt MS, Kwon J, Arshad MU, Sultan MT. Mangiferin: a natural miracle bioactive compound against lifestyle related disorders. Lipids Health Dis. 16(1), 84 (2017).

27. Sáyago-Ayerdi SG, Moreno-Hernández CL, Montalvo-González E et al. Mexican 'Ataulfo' mango (Mangifera indica L) as a source of hydrolyzable tannins. Analysis by MALDI-TOF/TOF MS. Food Res. Int. 51(1), 188-194 (2013).

28. Roberto J, De Souza R, Feitosa JPA et al. Spray-drying encapsulation of mangiferin using natural polymers. Food Hydrocoll. 33(1), 10-18 (2013).

29. Liu R, Liu Z, Zhang C, Zhang B. Gelucire44/14 as a novel absorption enhancer for drugs with different hydrophilicities: in vitro and in vivo improvement on transcorneal permeation. J. Pharm. Sci. 100(8), 3186-3195 (2011).

30. Rajendran P, Rengarajan T, Nandakumar N, Divya H, Nishigaki I. Mangiferin in cancer chemoprevention and treatment: pharmacokinetics and molecular targets. J. Recept. Signal Transduct. 35(1), 76-84 (2015).

- Uses this paper as reference because it shows the main therapeutic activities of mangiferin.

31. Telang M, Dhulap S, Mandhare A, Hirwani R. Therapeutic and cosmetic applications of mangiferin: a patent review. Expert Opin. Ther. Pat. 23(12), 1561-1580 (2013).

32. Peppas NA, Huang Y. Nanoscale technology of mucoadhesive interactions. Adv. Drug Deliv. Rev. 56(11), 1675-1687 (2004).

33. Deacon MP, McGurk S, Roberts CJ et al. Atomic force microscopy of gastric mucin and chitosan mucoadhesive systems. Biochem. J. 563, 557-563 (2000).

34. Choi EH, Man MQ, Wang F et al. Is endogenous glycerol a determinant of stratum corneum hydration in humans? J. Invest. Dermatol. 125(2), 288-293 (2005).

35. Björklund S, Engblom J, Thuresson K, Sparr E. Glycerol and urea can be used to increase skin permeability in reduced hydration conditions. Eur. J. Pharm. Sci. 50(5), 638-645 (2013).

36. Carrer V, Alonso C, Pont $\mathrm{M}$ et al. Effect of propylene glycol on the skin penetration of drugs. Arch. Dermatol. Res. doi:10.1007/s00403-019-02017-5 (2019) (Epub ahead of print).

37. Petrou G, Crouzier T. Mucins as multifunctional building blocks of biomaterials. Biomater. Sci. 6(9), 2282-2297 (2018).

38. Manconi M, Aparicio J, Vila M, Pendás J, Figueruelo J, Molina F. Viscoelastic properties of concentrated dispersions in water of soy lecithin. Colloids Surf. A Physicochem. Eng. Asp. 222(1), 141-145 (2003).

39. Manconi M, Marongiu F, Manca ML et al. Nanoincorporation of bioactive compounds from red grape pomaces: in vitro and ex vivo evaluation of antioxidant activity. Int. J. Pharm. 523(1), 159-166 (2017).

40. Caddeo C, Sales OD, Valenti D, Saurí AR, Fadda AM, Manconi M. Inhibition of skin inflammation in mice by diclofenac in vesicular carriers: liposomes, ethosomes and PEVs. Int. J. Pharm. 443(1-2), 128-136 (2013).

41. Dermatological and Transdermal Formulations. Walters KA. (Ed.). CRC Press, FL, USA (2019).

42. Manca ML, Valenti D, Sales OD, Nacher A, Fadda AM, Manconi M. Fabrication of polyelectrolyte multilayered vesicles as inhalable dry powder for lung administration of rifampicin. Int. J. Pharm. 472(1-2), 102-109 (2014).

43. Klemetsrud T, Jonassen H, Hiorth M, Kjøniksen AL, Smistad G. Studies on pectin-coated liposomes and their interaction with mucin. Colloids Surf. B Biointerfaces 103, 158-165 (2013).

44. Takeuchi H, Thongborisute J, Matsui Y, Sugihara H, Yamamoto H, Kawashima Y. Novel mucoadhesion tests for polymers and polymer-coated particles to design optimal mucoadhesive drug delivery systems. Adv. Drug Deliv. Rev. 57(11), 1583-1594 (2005).

45. Van Tran V, Moon J, Lee Y. Liposomes for delivery of antioxidants in cosmeceuticals: challenges and development strategies. J. Control. Rel. 300, 114-140 (2019). 
46. Benson HAE. Transfersomes for transdermal drug delivery. Expert Opin. Drug Deliv. 3(6), 727-737 (2006).

- Uses this paper as reference because it shows the properties of mucin and its use in nanomedicine.

47. Ahad A, Al-Saleh AA, Al-Mohizea AM et al. Formulation and characterization of Phospholipon $90 \mathrm{G}$ and Tween 80 based transfersomes for transdermal delivery of eprosartan mesylate. Pharm. Dev. Technol. 23(8), 787-793 (2018).

48. Larrucea E, Arellano A, Santoyo S, Ygartua P, Farmace Â. Combined effect of oleic acid and propylene glycol on the percutaneous penetration of tenoxicam and its retention in the skin. Eur. J. Pharm. Biopharm. 52, 113-119 (2001).

49. Pedersen LK, Jemec GBE. Plasticising effect of water and glycerin on human skin in vivo. J. Dermatol. Sci. 19(1), 48-52 (1999).

50. Bansil R, Turner BS. Mucin structure, aggregation, physiological functions and biomedical applications. Curr. Opin. Colloid Interface Sci. 11(2-3), 164-170 (2006).

51. Karewicz A, Bielska D, Gzyl-Malcher B, Kepczynski M, Lach R, Nowakowska M. Interaction of curcumin with lipid monolayers and liposomal bilayers. Colloids Surf. B Biointerfaces 88(1), 231-239 (2011).

52. Boink MA, Van Den Broek LJ, Roffel S, Nazmi K. Different wound healing properties of dermis, adipose, and gingiva mesenchymal stromal cells. Wound Repair Regen. 24(1), 100-109 (2016).

53. Mauricio P, Luisa M, Herrera-ruiz M, Zamilpa A, Jiménez-ferrer E, Trejo-tapia G. In vivo anti-inflammatory and anti-ulcerogenic activities of extracts from wild growing and in vitro plants of Castilleja tenuiflora Benth. (Orobanchaceae). J. Ethnopharmacol. 150(3), 1032-1037 (2013).

54. Klein-Szanto AJP, Conti CJ, Aldaz CM, Clapp N, Nesnow S, Slaga TJ. Effects of chronical topical application of 12-O-tetradecanoylphorbol-13-acetate on the skin and internal organs of SENCAR mice. Environ. Health Perspect. 68, 75-80 (1986). 